\title{
LES MOTS DU CORPS DANS LE LANGAGE DE GEORGES BRASSENS
}

(« Vos cours d'anatomie allez les prendre ailleurs. $\left.{ }^{1} »\right)$

\author{
Dávid SzABÓ \\ Université Eötvös Loránd (ELTE), Budapest
}

\begin{abstract}
En): The paper deals with the way French poet, songwriter and singer Georges Brassens (1921-1981) speaks about the human body. The analysis of stylistic variation in his works has been based on the classification of two dictionaries: Garitte's Mais où sont les mots d'antan? (2017) and Le Petit Robert 2018, and on a questionnaire survey carried out on the Internet among 99 native speakers of French. According to this study the relevant vocabulary consists of approximately two thirds of nonstandard (colloquial, slang etc.) and one third of standard (formal, literary) items. The last category (littéraire in French) seems particularly interesting as it can be subject to different interpretations. The analysis of a particular song (Vénus Callipyge) also shows us that Brassens does not always want to be explicit when speaking about the human body.
\end{abstract}

Keywords (En): Georges Brassens; literary register; registers; stylistic variation

Mots-clés (Fr) : Georges Brassens ; littéraire ; niveaux de langue ; registres ; variation stylistique

DOI : $10.32725 /$ eer.2021.012

\section{Introduction}

Dans ce travail, nous allons étudier les mots et expressions relatifs au corps humain dans le langage de Georges Brassens, indiscutablement un des chanteurs et poètes français les plus importants au $\mathrm{XX}^{\mathrm{e}}$ siècle $^{2}$. Ce qui nous intéressera essentiellement dans les textes du chansonnier, c'est la variation stylistique qui caractérise la façon de nommer les parties du corps humain dans ses chansons, autrement dit, les registres et niveaux de langue spécifiques aux choix lexicaux de Brassens dans ce domaine qui lui était particulièrement $\mathrm{cher}^{3}$. Nous nous concentrerons surtout sur les éléments langagiers qui se distinguent de l'usuel, du stylistiquement neutre, que ce soit du côté du non-conventionnel, du non-standard, ou, au contraire, du côté des registres soutenu ou littéraire ${ }^{4}$. Ce qui implique que malgré tous nos regrets, une chanson remarquable comme Rien à jeter (BRASSENS, 1993 : 183-185), que nous citons juste après le titre de ce travail et qui est presque

\footnotetext{
${ }^{1}$ BRASSENS (1993 : 184).

2 «Brassens [...] n'a cessé de rêver poésie tout en martelant qu'il n'écrivait que des chansons », fait remarquer Perraud (2001 : 74). Pourtant, García Márquez n’a pas peur de le considérer comme le plus grand poète français de son temps : c'est Garitte (2017) qui le cite, en quatrième de couverture de son dictionnaire.

${ }^{3}$ Pour appuyer notre constatation, il suffit de mentionner ici des chansons célèbres comme Le nombril des femmes d'agents, Les sabots d'Hélène, Vénus Callipyge, La fessée, Rien à jeter ou Le blason, toutes consacrées, du moins partiellement, à certaines parties du corps... de la femme.

${ }^{4}$ Par littéraire nous comprendrons ici un emploi créatif, artistique du langage, ce qui implique une catégorie bien plus vaste que celle de « la langue écrite élégante » (REY, REY-DEBOVE, 2018 : XXXI). Cf. aussi l'article de KovÁCs (2020) sur ce sujet.
}

$$
-141-
$$


entièrement consacrée à la description du corps de la «mie » du poète, a peu d'intérêt du point de vue de cette analyse, car les parties du corps de la belle y sont généralement nommées d'une manière tout à fait usuelle: cheveux, joues, hanches...

Pour l'élaboration de notre corpus, nous nous sommes servi du dictionnaire de Garitte (2017) Brassens. Mais où sont les mots d'antan?, ainsi que de l'intégrale de l'œuvre de Georges Brassens, Les Chansons d'abord (BRASSENS, 1993). L'étude du corpus a été complétée par une enquête par questionnaires diffusés via Internet.

Dans un travail récent (SZABÓ, 2021), nous avons étudié les mots de l'amour (physique) dans les œuvres de Brassens. Cependant, nous n'y avons pas tenu compte de ces accessoires inévitables de l'amour physique que sont les parties génitales et dont les noms semblent constituer également un sujet très intéressant. Il est temps de nous rattraper. Par contre, dans ce précédent travail, nous avons recensé bon nombre d'expressions qui ne désignent pas des parties du corps humain, mais dans lesquelles des mots du corps jouent un rôle important :

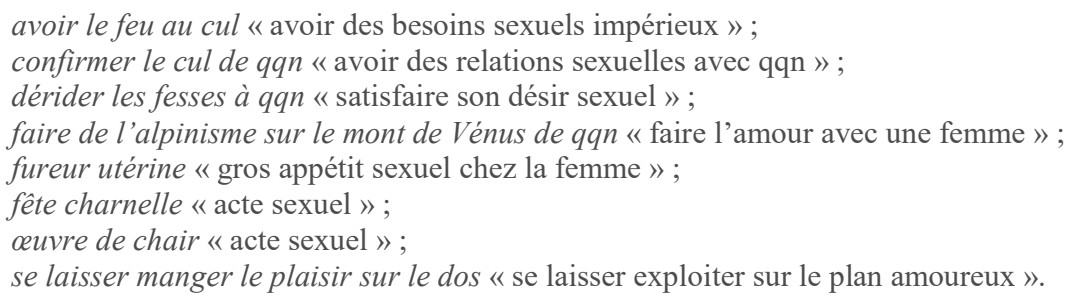

D'après les conclusions de notre travail sur les mots de l'amour chez Brassens, nous pouvons formuler l'hypothèse selon laquelle dans le domaine des mots du corps humain, nous devrions aussi nous attendre à une mixité stylistique : des registres non standard (familier, populaire) mélangés à du littéraire (dans le sens de registre - souvent mais pas toujours - soutenu de la création littéraire) mais aussi à une pauvreté inattendue ${ }^{5}$ en argotismes ou mots obscènes ${ }^{6}$.

\section{Les mots du corps chez Brassens}

Dans ce chapitre, nous présenterons les noms des parties du corps tels que Brassens les utilise dans ces chansons, en fonction de leur registre. Comme indiqué dans l'introduction, les mots et expressions usuels seront exclus de l'analyse, ainsi que les éléments familiers les plus courants (par ex., cul, fesses). Dans la sélection des termes spécifiques au langage de Brassens, ainsi que dans l'identification des nuances stylistiques, nous nous sommes appuyé avant tout sur le dictionnaire Brassens de GARITTE (2017), dont le dépouillement a été complété par des vérifications dans BRASSENS (1993) et Le Petit Robert 2018 (REY-DEBOVE, 2017).

\footnotetext{
${ }^{5}$ Compte tenu des interdictions ayant frappé l'auteur du Gorille et de sa «mauvaise » réputation de « pornographe du phonographe » (BRASSENS, 1993 : 93-96).

${ }^{6} \mathrm{Cf}$. aussi Perraud (2001 : 75).
} 


\section{Mots et expressions familiers :}

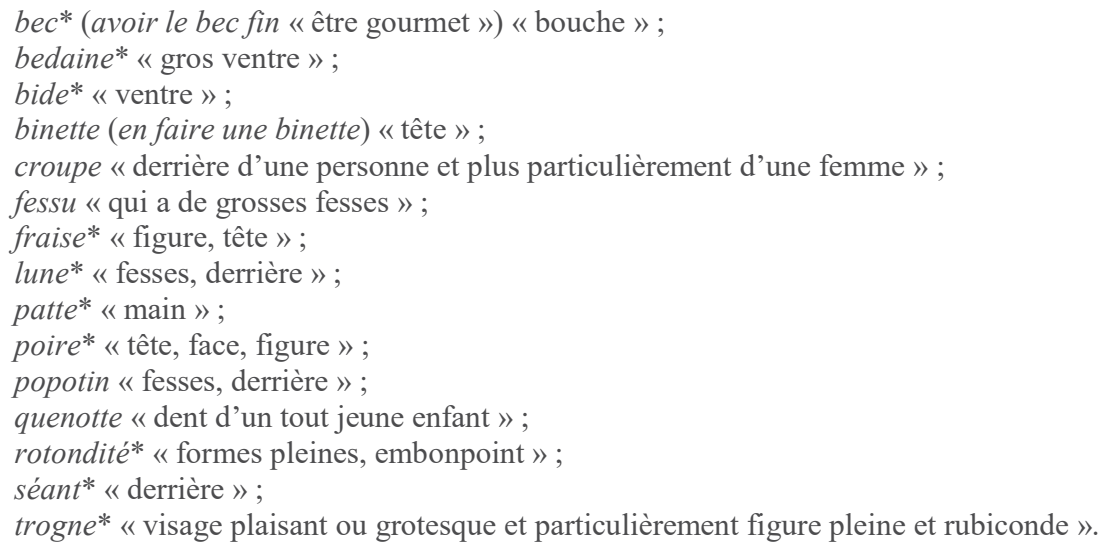

Les mots suivis d'un astérisque ne sont marqués comme familiers que dans Le Petit Robert. Notons que frimousse «visage agréable» est considéré comme familier par GARITTE (2017) mais ne reçoit aucune mention dans Le Petit Robert. Parmi les éléments familiers, académie « corps (nu) » est accompagné de la mention vieilli dans les deux dictionnaires.

\section{Mots et expressions populaires (?) :}

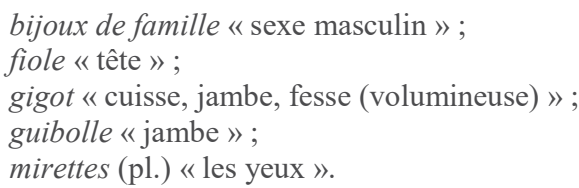

Le point d'interrogation après le terme populaire s'explique par le fait que les éléments lexicaux ci-dessus sont classés dans la catégorie populaire par Garitte, et non par Le Petit Robert qui les qualifie systématiquement de familiers. La raison de cela est sans aucun doute la disparition progressive de la mention pop. d'un certain nombre de dictionnaires parmi lesquels Le Petit Robert ${ }^{7}$.

Pour rendre la complexité stylistique de certains mots, il faut ajouter que chez Garitte, fiole est accompagné de la mention vieilli, et gigot de l'explication «par plaisanterie $»$.

\footnotetext{
${ }^{7}$ À titre d'exemple, mentionnons ici les dictionnaires bilingues de Jean PERROT (2003) (Magyarfrancia kéziszótár - Dictionnaire hongrois-français, Szeged, Grimm Kiadó) et de Vilmos BÁRDOSI et Dávid SzABó (2007) (Francia-magyar kéziszótár - Dictionnaire français-hongrois, Budapest, Akadémiai Kiadó) dans lesquels la mention pop. est absente. Ce phénomène s'explique en partie par la constatation que GADET (1992 : 27) formule ainsi : « La dénomination de français populaire est très peu satisfaisante... ».
} 


\title{
1.3. Mots et expressions argotiques :
}

\author{
fuseaux (pl.) «jambes »; \\ louche « main »; \\ miches (pl.) «postérieur»; \\ valseur « postérieur (surtout féminin)».
}

Les argotismes sont donc rares et deux d'entre eux - louche et miches - sont qualifiés de familiers par Le Petit Robert, alors que les deux autres (fuseaux et valseur) y sont absents, mais figurent dans le dictionnaire d'argot de COLIN et al. (1990). Néanmoins, malgré la rareté et l'aspect problématique des éléments argotiques et populaires, nous pouvons conclure qu'avec les termes familiers les mots et expressions non standard ou non conventionnels constituent une large majorité parmi les éléments stylistiquement marqués du corpus.

\subsection{Littéraire et autres marques d'usage}

Il y a aussi des termes qui sont étiquetés comme littéraires chez Garitte : appas ${ }^{8}$ (pl.) "attraits d'une femme", chef $^{9}$ " tête" et gorge "seins de la femme». Cependant, Le Petit Robert considère le premier comme vieilli ou plaisant, le deuxième simplement comme vieilli.

Bien qu'il ne s'agisse pas toujours de registres ou de niveaux de langue proprement dits, certains mots du corps identifiés dans les paroles de Brassens sont accompagnés dans Le Petit Robert de marques d'usage pouvant indiquer des conséquences stylistiques comme anat. (terme d'anatomie) dans le cas de Mont de Vénus « pubis, chez la femme » ou éminence qui figure dans l'expression éminence charnue " postérieur renflé ». Callipyge " aux belles fesses ${ }^{10}$ » s'accompagne de la mention didactique dans Le Petit Robert, attribut signifie spécialement, c'est-à-dire dans un sens plus étroit, " organes sexuels mâles » dans l'expression attributs virils, tandis que la locution organes procréateurs («organes génitaux ») est présentée comme vieillie ou plaisante.

\section{Une enquête par questionnaires}

Ce que nous trouvions le plus intéressant, c'est que dans le corpus des mots du corps chez Brassens, il y avait de nombreux mots et expressions qui n'étaient accompagnés d'aucune mention dans les deux dictionnaires consultés (GARITTE, 2017 ; Le Petit Robert 2018) mais qui ne nous semblaient pas tout à fait usuels, autrement dit neutres dans le sens stylistique du terme ${ }^{11}$. Nous pensons ici à des termes comme accroche-cœeur « mèche de cheveux en croc, collée sur la tempe »,

\footnotetext{
${ }^{8}$ Ce n'est pas un mot du corps mais un mot relatif au corps.

${ }^{9}$ Chez Garitte, le mot est également accompagné de la mention classique.

${ }^{10}$ GARITTE (2017) ne retient que ce sens, mais l'autre signification présente dans Le Petit Robert (« qui a les fesses exagérément développées ») semble aussi caractériser le mot tel qu'il est employé, comme nous allons voir par la suite, dans la chanson Vénus Callipyge (BRASSENS, 1993 : 148-149).

${ }^{11}$ Certaines de ces expressions comme les choses «testicules» ou valseur "postérieur » étaient absentes du Petit Robert.
} 
bajoue "grosse joue humaine, pendante, fortement prononcée », balafré "marqué d'une cicatrice, particulièrement au visage » ou con « sexe de la femme ». Certaines expressions non marquées chez Garitte n'étaient que partiellement caractérisées ${ }^{12}$ dans Le Petit Robert: c'était notamment le cas d'éminence charnue ou d'organes procréateurs. Parfois, c'était la marque d'usage réservée à une expression par le dictionnaire qui ne semblait pas bien caractériser l'utilisation du terme chez Brassens (par ex., mont de Vénus) ${ }^{13}$.

Pour y voir plus clair, autrement dit pour vérifier si nos intuitions quant aux nuances stylistiques de ces mots et expressions étaient justes, nous avons conduit une enquête par questionnaires sur les nuances stylistiques de 20 mots et expressions non marqués dans GARITTE (2017). Les questionnaires ont été diffusés sur Internet par le biais de Google Forms.

Après des questions sur l'âge, le sexe et le pays d'origine de l'enquêté, les informateurs devaient indiquer le registre de vingt mots et expressions relatifs au corps humain. Ils avaient le choix entre les catégories argotique, familier, usuel, soutenu, littéraire et autre.

Dans l'état de la recherche, 99 personnes ont rempli le questionnaire : 54,5\% de femmes et $45,5 \%$ d'hommes. Leur âge variait entre 20 et 76 ans : 21 informateurs étaient âgés d'entre 20 et 35 ans, 51 d'entre 36 et 59 ans et 27 avaient 60 ans ou plus. 94 enquêtés sur 99 étaient français, trois étaient belges, et il y avait aussi un informateur d'origine portugaise et un d'origine turque.

Bien sûr, la plupart des enquêtés n'avait sans doute pas fait d'études de linguistique ou de stylistique, alors que même pour des spécialistes, ce type d'exercice est loin d'être facile. Mais en comptant sur la loi des grands nombres, on peut espérer à juste titre que l'opinion d'une centaine de locuteurs natifs pourrait refléter assez fidèlement les jugements stylistiques souvent inconscients du public francophone ${ }^{14}$.

La question la plus intéressante était naturellement la vérification du bien-fondé d'un jugement d'ordre linguistique : celui de la neutralité stylistique (selon Garitte et en partie Le Petit Robert) d'une vingtaine de mots et expressions du corps qui apparaissent dans l'œuvre de Brassens.

L'enquête semble avoir donné raison à nos interrogations. Seulement quatre sur vingt termes ont été caractérisés par nos informateurs comme usuels. En disant « caractérisé comme tel », nous pensons à la catégorie la plus fréquemment choisie par les enquêtés, ce qui ne signifie pas toujours la majorité absolue des réponses. $67,7 \%$ ont marqué joufflu « qui a de grosses joues » comme usuel, ce chiffre étant

${ }^{12}$ Le dictionnaire ne caractérisait que certains éléments d'une locution et non la locution dans son intégralité.

${ }^{13}$ Nous avons aussi ajouté à la liste une locution non marquée chez Garitte et caractérisée comme familière par Le Petit Robert : gras du bide.

${ }^{14} \mathrm{Il}$ y a un facteur particulièrement important que nous ne devons pas ignorer, celui du temps. La majeure partie de l'œuvre de Brassens remonte aux années 1950-70, alors que notre enquête a été menée en 2021. Près d'un tiers de nos informateurs (essentiellement les plus de 60 ans) ont pu « connaître » Brassens de son vivant, tandis que pour les 20-35 ans, il représente probablement une figure lointaine. Mais il ne faut pas oublier que le point de départ de notre enquête est la classification d'un dictionnaire (celui de Garitte) publié récemment, en 2017 ... et que la survie d'un auteur ne peut être possible qu'à travers les jugements d'un public postérieur. 
de $58,6 \%$ dans le cas de balafré «marqué d'une cicatrice », de 44,4 \% dans le cas de bajoue "grosse joue humaine » et seulement de 29,3\% dans le cas de toupet "mèches de cheveux relevées au-dessus du front». La figure 1 illustre bien la complexité des jugements stylistiques relatifs à un mot donné : plus de $70 \%$ n'étaient pas d'accord avec la catégorie usuel (qui était pourtant le premier choix des informateurs) et une majorité absolue $(51,5 \%)$ semblait préférer littéraire ou soutenu.

\section{2. toupet (mèches de cheveux relevées au-dessus du front)}

99 réponses

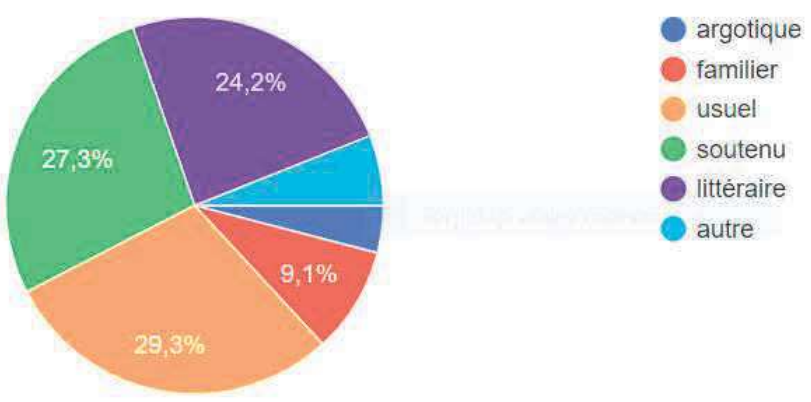

Figure 1 - Jugements stylistiques relatifs au mot toupet.

Nos informateurs n'ont identifié que deux argotismes : valseur "postérieur » avec $72,7 \%$ et con « sexe de la femme » avec 55,6 \% (Fig. 2) ${ }^{15}$. Cette pauvreté en argot semble confirmer une de nos hypothèses de travail (voir aussi PERRAUD, 2001 : 75). Par contre, les éléments familiers (au nombre de quatre) sont plus nombreux: gras $d u$ bide "ventripotent» $(62,6 \%)$, menotte «petite main» $(49,5 \%)$, les choses « testicules » $(32,3 \%)$, membre de ma famille « membre viril » $(30,3 \%)$. On peut constater que les éléments non standard (familiers, argotiques, populaires) constituent à peu près les deux tiers des mots du corps chez Brassens.

\footnotetext{
${ }^{15}$ Si vous pensez (comme Le Petit Robert) que les mots tabous très anciens comme con ne sont pas particulièrement argotiques, nous sommes d'accord. Cependant, outre le respect dû aux résultats d'une enquête, n'oublions pas que le mot figure aussi dans le dictionnaire d'argot de COLIN et al. (1990).
} 


\section{5. con (sexe de la femme)}

99 réponses

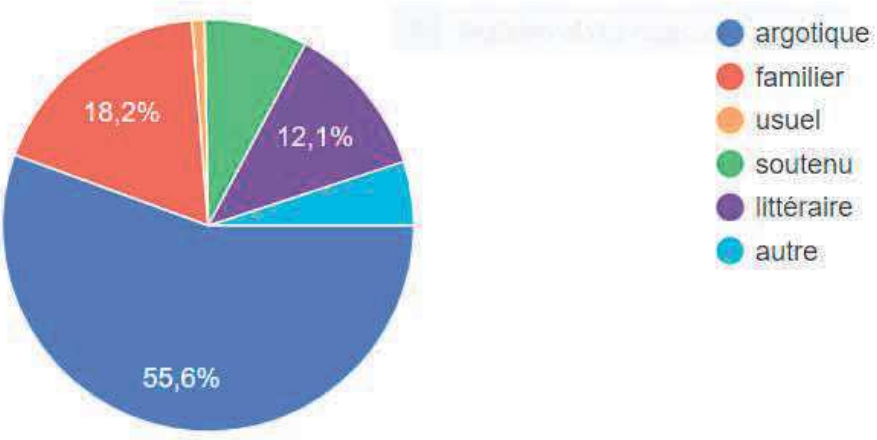

Figure 2 - Jugements stylistiques relatifs au mot con.

Ce sont les registres soutenu et littéraire qui ont été choisis par nos informateurs avec la plus grande fréquence. Galbe « contours du corps » (44,4\%), attributs virils « organes sexuels mâles » $(40,4 \%)$, organes procréateurs « organes génitaux » $(39,4 \%)$, phallus « membre viril en érection » $(37,4 \%)$ et lippe «lèvre inférieure épaisse » $(30,3 \%)$ sont majoritairement qualifiés de soutenus, tandis que mont de Vénus «pubis chez la femme» $(72,7 \%)$, éminence charnue «fesses renflées » (51,5\%), l'endroit où le dos ressemble à la lune « postérieur » $(50,5 \%)$, accrochecœur " mèche de cheveux en croc » $(44,4 \%)$ et toison "chevelure abondante " $(38,4 \%)$ sont avant tout marqués comme littéraires.

Il peut sembler naturel d'analyser ensemble les termes soutenus et littéraires, étant donné que Le Petit Robert, comme nous l'avons vu ci-dessus, définit littéraire comme « qui n'est pas d'usage familier, qui s'emploie surtout dans la langue écrite élégante ». À propos de soutenu (qui ne figure pas comme marque d'usage dans le dictionnaire en question), nous lisons dans Le Petit Robert : «Qui se maintient à un certain niveau de pureté, d'élégance, évite toute familiarité ». En dehors de l'insistance sur l'écrit dans le cas de littéraire, les deux définitions sont quasiment identiques et s'opposent à celles des registres non standard.

Il est important d'insister ici sur une autre possibilité d'interprétation du terme littéraire. Alors que des mots comme galbe ou lippe, une fois écrits, semblent pouvoir correspondre tout aussi bien à la définition du littéraire citée plus haut qu'à celle du soutenu, une expression comme l'endroit où le dos ressemble à la lune (Fig. 3), sans être particulièrement soutenue, serait littéraire dans le sens de langage spécifique à la création littéraire ${ }^{16}$.

${ }^{16}$ Ce problème s'explique en partie par le fait que l'usage linguistique et lexicographique diffère parfois en ce qui concerne l'identification des niveaux de langues ou registres : par exemple, DuBOIS et al. $(1989: 337,417)$ ne distinguent pas de niveau ou de registre littéraire, tandis que Le Petit Robert n'a pas de mention soutenu, mais utilise la catégorie littéraire. Cf. aussi l'article de KovÁCs (2020) sur ce sujet. 


\section{7. l'endroit oủ le dos ressemble à la lune (postérieur)}

99 réponses

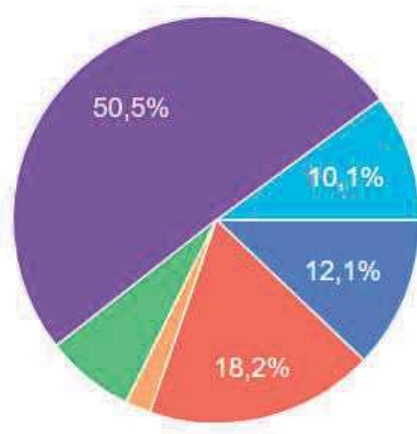

Figure 3 - Jugements stylistiques relatifs à l'expression l'endroit où le dos ressemble à la lune.

\section{Des expressions figées ou défigées}

Un des aspects les plus caractéristiques de l'œuvre de Brassens est constitué par le grand nombre de locutions figées qu'on trouve dans ses textes. Cependant, ce qui semble la caractériser encore davantage, c'est le défigement de locutions bien connues (GARITTE, 2017 : 17-18).

Nous avons inclus dans notre analyse des mots du corps chez Brassens des expressions comme bijoux de famille ou mont de Vénus, etc., mais la grande majorité des unités phraséologiques relevées dans notre corpus ne désigne pas des parties du corps humain, mais se sert des mots du corps pour aboutir à des significations souvent très éloignées de la description de notre anatomie. Ainsi, ces expressions (plus ou moins figées) ne semblent relever que marginalement de notre thématique. Pour ne donner que quelques exemples :

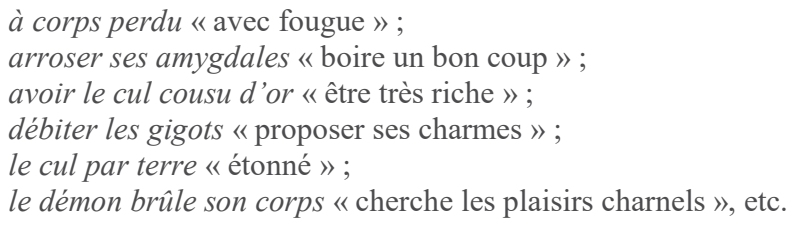

De nombreuses locutions défigées comportent également des mots du corps sans qu'elles puissent être considérées comme des mots ou expressions du corps proprement dits. Il s'agit donc ici d'un thème de recherche particulièrement intéressant mais qui ne fait pas réellement partie de notre analyse :

avoir à cour de mourir plus haut que son cul cf. « péter plus haut que son cul »; avoir une fesse qui dit merde à l'autre cf. " avoir un œil qui dit merde à l'autre »; chercher des poux dans la tonsure cf. « chercher des poux à qqn"; recevoir à bras fermés $\mathrm{cf}$. « recevoir à bras ouverts »; 

tué »;

vendre les génitoires de l'ours un peu trop tôt $\mathrm{cf}$. « vendre la peau de l'ours avant de l'avoir voir votre académie et puis mourir cf. « voir Naples et puis mourir », etc.

\section{Une chanson consacrée à une partie du corps : Vénus Callipyge $e^{17}$}

Nous terminerons notre étude des mots du corps dans les chansons de Brassens par une brève analyse d'une des œuvres du chansonnier-poète consacrées à une partie du corps en particulier : comme nous l'apprenons dans Le Petit Robert, -pyge vient du grec pugê «fesse»; et la chanson parle en effet de cette partie du corps humain, plus particulièrement du corps de la femme.

L'adjectif qui figure dans le titre de la chanson signifie selon GARITTE (2017) « aux belles fesses », ce qui est confirmé par le premier vers de la deuxième strophe : "Votre dos perd son nom avec si bonne grâce ». Cependant, l'autre sens de callipyge («qui a les fesses exagérément développées » selon Le Petit Robert qui qualifie le mot de didactique) est également présent chez Brassens qui parle notamment de « volume étonnant».

Le texte abonde en manières indirectes de nommer (par allusion) la partie du corps en question : le dos qui perd son nom et le volume étonnant mentionnés cidessus, l'expression «en embrassant Fanny $»^{18}$, le mot galbe caractérisé comme soutenu par nos enquêtés, les pronoms le mien et le vôtre $e^{19}$, le fait de placer l'orgueil un peu bas, la demande de ne pas s'asseoir ou la mention ironique de la loi de la pesanteur renvoient sans exception, et sans la nommer explicitement, à cette région de l'anatomie humaine ${ }^{20}$.

Cependant, Brassens - qui utilise aussi le mot académie (qualifié de familier et vieilli par les dictionnaires) dans sa chanson - n'a pas peur d'appeler un chat un chat. Pour parler de la partie du corps susnommée, il se sert aussi des termes bien plus courants fesses et cul. Ce dernier fait son apparition dans la locution faux cul (caractérisé comme familier par Garitte et Le Petit Robert) laquelle, dans le contexte et contrairement à l'interprétation de GARITTE (2017: 457), ne signifie pas uniquement «hypocrite », mais aussi « rembourrage porté sous la robe, au bas du dos » selon la définition du Petit Robert.

L'analyse de cette chanson consacrée à un sujet tabou montre bien que malgré sa «mauvaise réputation» (cf. MÉRIGAUD, 2001: 58-75), Brassens n'a pratiquement jamais été grossier et savait se montrer, à l'occasion, particulièrement pudique $^{21}$.

\footnotetext{
${ }^{17}$ Pour le texte intégral de cette chanson publiée en 1965 voir BRASSENS (1993: 148-149).

${ }^{18}$ Il s'agit ici d'une tradition suivant laquelle le joueur qui perdait au jeu de boules devait embrasser un panneau en bois représentant une femme exhibant son derrière (GARITTE, $2017: 428$ ).

${ }^{19}$ Ces pronoms possessifs font référence à une chanson paillarde (Le Duc de Bordeaux) et doivent être compris comme une allusion à la partie du corps en question (GARITTE, 2017: 695; voir aussi https://xavier.hubaut.info/paillardes/texte.htm. Consulté le 11.02.2021).

${ }^{20}$ N'oublions pas que le choix du titre, avec un adjectif didactique construit d'éléments d'origine grecque, constitue en soi une façon de cacher la partie du corps en question.

${ }^{21}$ Naturellement, cette pudeur est contrebalancée par le choix osé du thème de la chanson, ainsi que par certaines allusions, par ex., celle à une chanson paillarde.
} 


\section{En guise de conclusion}

Dans ce travail, nous avons analysé les mots du corps dans l'œuvre de Georges Brassens du point de vue de la variation stylistique. Notre étude reposait essentiellement sur les marques d'usage qui accompagnent les éléments lexicaux de notre corpus dans le dictionnaire Brassens de GARITTE (2017) et dans Le Petit Robert 2018. Étant donné qu'un nombre relativement important de mots et expressions n'avait reçu aucune mention particulière dans les deux dictionnaires, nous avons complété l'analyse lexicographique par une enquête par questionnaires menée via Internet auprès d'une centaine de locuteurs francophones natifs. Cette enquête semble avoir donné raison à nos interrogations : 15 sur 19 termes dépourvus de marques d'usage dans les dictionnaires ont été caractérisés comme littéraires, soutenus, familiers et (parfois) argotiques par nos informateurs.

D'après notre analyse, le vocabulaire que Brassens emploie pour parler du corps humain se partage entre éléments lexicaux standard et non standard, avec néanmoins une préférence pour la seconde catégorie: compte tenu des jugements des dictionnaires et de nos enquêtés, près des deux tiers de notre corpus peuvent être considérés comme non standard (qualifiés avant tout de familiers mais aussi de populaires ou argotiques) et plus d'un tiers appartient au standard (littéraire, soutenu ou usuel dans la plupart des cas) ${ }^{22}$.

C'est le niveau ou registre littéraire qui semble mériter ici une attention particulière. Selon une certaine tradition lexicographique et notamment la définition du Petit Robert (voir plus haut), ce terme caractérise un usage soutenu et écrit de la langue et s'oppose notamment au registre familier. Cependant, certaines des expressions en question ne semblent pas particulièrement soutenues mais témoignent plutôt de la créativité artistique de leur auteur ${ }^{23}$ : nous pensons par exemple à des expressions comme l'endroit où le dos ressemble à la lune ${ }^{24}$.

Nous avons terminé notre analyse par l'étude des mots du corps dans Vénus Callipyge, une chanson consacrée à une partie du corps en particulier. Cette brève analyse montre bien que ce « pornographe ${ }^{25}$ » autoproclamé qu'était Brassens n'est pratiquement jamais grossier ni vulgaire et choisit parfois de parler de certaines parties du corps sans les nommer. Celui que Merigaud (2001:3) appelle poète pudique et qui, selon la chanson, ne faisait « voir [s]es organes procréateurs à personne, excepté [s]es femmes et [s]es docteurs» (BRASSENS, 1993: 128), préférait dans certains cas parler du corps humain sans être très explicite.

\footnotetext{
${ }^{22}$ Il faut rappeler ici que les termes courants et neutres comme cheveux, hanche, sein ou pied - qui appartiennent naturellement au standard et sont fréquents dans les textes de Brassens - étaient exclus de notre analyse.

${ }^{23}$ La redéfinition de littéraire comme langage de la création littéraire se rapproche de l'emploi sociolinguistique du terme registre comme "variété linguistique appropriée à une situation sociale particulière » (MOREAU, 1997 : 238).

${ }^{24}$ Notons ici que contrairement à la majorité des termes de notre corpus largement attestés ailleurs, l'endroit où le dos ressemble à la lune pourrait faire partie des créations propres à Brassens et ainsi devrait être considéré comme un hapax.

${ }^{25}$ Voir la chanson Le pornographe (BRASSENS, 1993 : 93-96).
} 


\section{BIBLIOGRAPHIE}

BRASSENS Georges (1993), Les Chansons d'abord, Paris, Librairie Générale Française.

COLIN Jean-Paul, MÉvel Jean-Pierre, LECLÈRE Christian (1990), Dictionnaire de l'argot, Paris, Larousse.

DuboIS Jean, GIACOMO Mathée, GUESPIN Louis, MARCELlESI Christiane, MARCELleSI Jean-Baptiste, MÉVEL Jean-Pierre (1989), Dictionnaire de linguistique, Paris, Larousse.

GADET Françoise (1992), Le français populaire, Paris, Presses Universitaires de France.

GARITTE Jean-Louis (2017), Brassens. Mais où sont les mots d'antan?, Neuilly, Atlande.

KovÁCs Máté (2020), Vers une redéfinition du registre littéraire : Les variétés de langue non standard comme langue de la narration littéraire, in : MUDROCHOVÁ Radka, Courbon Bruno (éds.), Diversité et variations de la langue française au XXI siècle, Plzeň, NAVA, p. 261-274.

MÉRIGAUD Bernard (éd.) (2001 [sans date]), Brassens. Un copain d'abord, Télérama hors-série, Paris, nouvelle édition.

Moreau Marie-Louise (1997), Sociolinguistique. Concepts de base, Sprimont, Mardaga.

PERRAUD Antoine (2001 [sans date], La langue fourchue, in : MÉRIGAUD Bernard (éd.), Brassens. Un copain d'abord, Télérama hors-série, Paris, nouvelle édition, p. 74-75.

ReY Alain, ReY-Debove Josette (éds.) (2017), Le Petit Robert de la langue française, Paris, Dictionnaires Le Robert - SEJER, Nouvelle édition millésime 2018.

SzABÓ Dávid (2021), «Parlez-moi d'amour (et j’vous fous mon poing sur la gueule)». Les mots de l'amour physique chez Georges Brassens, Acta Universitatis Lodziensis. Folia Litteraria Romanica 16, Łódź, p. 241-249. 